\title{
Powder keg divisions in the critical state regime: transition from continuous to explosive percolation
}

\author{
Zongzheng Zhou and Antoinette Tordesillas * \\ School of Mathematics and Statistics, University of Melbourne, 3010, Australia
}

\begin{abstract}
The underlying microstructure and dynamics of a dense granular material as it evolves towards the "critical state", a limit state in which the system deforms with an essentially constant volume and stress ratio, remains widely debated in the micromechanics of granular media community. Strain localization, a common mechanism in the large strain regime, further complicates the characterization of this limit state. Here we revisit the evolution to this limit state within the framework of modern percolation theory. Attention is paid to motion transfer: in this context, percolation translates to the emergence of a large-scale connectivity in graphs that embody information on individual grain displacements. We construct each graph $G(r)$ by connecting nodes, representing the grains, within a distance $r$ in the displacement-state-space. As $r$ increases, we observe a percolation transition on $G(r)$. The size of the jump discontinuity increases in the lead up to failure, indicating that the nature of percolation transition changes from continuous to explosive. We attribute this to the emergence of collective motion, which manifests in increasingly isolated communities in $G(r)$. At the limit state, where the jump discontinuity is highest and invariant across the different unjamming cycles (drops in stress ratio), $G(r)$ encapsulates multiple kinematically distinct communities that are mediated by nodes corresponding to those grains in the shear band. This finding casts light on the dual and opposing roles of the shear band: a mechanism that creates powder keg divisions in the sample, while simultaneously acting as a mechanical link that transfers motion through such subdivisions moving in relative rigid-body motion.
\end{abstract}

\section{Introduction}

Percolation has profound implications for many disordered complex systems in social environments, Nature and technology: e.g., outbreak of an epidemic disease, social upheaval, spread of fire in a forest, computer virus or malware on computer networks, and power grid failure (see [1-4], and references therein). In multiphase granular and soft matter systems, percolation has been studied extensively in the context of fluid transport and applied in various industrial processes, including oil and gas recovery, dewatering, and in the manufacture of concrete, slurries and pastes $[5,6]$. In this context, the study of percolation concerns itself with a nontrivial critical behavior or phase transition that manifests as a sudden onset of large-scale connectivity in the system.

In 2009, Achlioptas and co-workers introduced a new class of percolation phenomena, coined explosive percolation [7]. Compared with ordinary percolation where the transition is continuous, explosive percolation is marked by an abrupt jump or discontinuous transition to a largescale connectivity through steps that are much smaller than the system size. Though still in its infancy [4], explosive percolation has already attracted enormous interest with some applications, albeit still few, to finite real-world networks. For example, it has been shown that monitoring

\footnotetext{
^e-mail: atordesi@unimelb.edu.au
}

the explosive percolation process on modular networks is useful for understanding the underlying community structure of proteins [2] and social networks [3]. The basic idea behind these real networks is that an initially disordered system can suddenly become ordered through clustering behavior; interacting units of a complex system, which behave in similar ways, form clusters or cliques. In the representative networks where the nodes are the units, such clustering behavior manifest as "network communities", which are partitions of graphs into groups of nodes with high levels of intra-connections and relatively sparse interconnections.

Here, we search for similar signatures of critical behavior in the "critical state regime" where distinct patterns in motion are widely observed. Take, for instance, samples undergoing localized failure. In the transition to this limit state, the sample suddenly splits into subdomains that move in relative rigid-body motion, while mediated by shear bands rich in vortices [8]. Once in the limit state, these "powder keg" divisions in kinematics intermittently form across unjamming cycles (drops in stress ratio), in between periods of relatively quiescent jamming cycles (rises in stress ratio). Each such unjamming cycle is triggered by collective force chain failure by buckling inside the shear band, a mechanism that causes adjacent subdomains on either side of the band to unload elastically while undergoing relative rigid-body motion. These 
failure events, accompanied by bursts to a peak in dissipation rate, kinetic energy and nonaffine deformation at the macroscale, have been widely observed in discrete element simulations [9].

Given the rich mechanisms and patterns in the limit state, our objective in this study is twofold: (a) to uncover a possible percolation transition in the dynamics of a dense granular material in the limit state, and (b) to use this critical percolation (if it exists) to gain new insights into the significance of self-organized, collective motions that characterize this limit state. To this end, we examine the percolation process in graphs embodying information on individual grain motions. Our data set is from a discrete element simulation of a biaxial compression test in which the sample deforms in the presence of strain localization in the limit state. We study the emergence of a large-scale connectivity in graphs $G(r)$ where the nodes are the grains; nodes close to each other in the displacement-state-space (i.e., within a distance $r$ ) are preferentially connected by links. As we tune the parameter $r$ from small to large, a percolation transition characterised by the size of the largest connected component can be observed. We study the nature of this percolation transition and attempt to relate the evolution of the critical point for percolation transition to the observed cycles of jamming-unjamming dynamics as evident in the rises and falls in the stress ratio.

The remainder of this paper is organized as follows. We describe the data set analysed in Section 2 and the method in Section 3. Key results are discussed in Section 4 before the concluding summary in Section 5.

\section{Data}

The data set comes from a well-studied simulation of 5098 spherical grains submitted to planar biaxial compression under constant confining pressure [9]. This simulation, which is from a model developed using the discrete element method (DEM), has been described elsewhere and many facets of it have been reported in the literature (e.g., $[9,10])$. It incorporates a contact moment (or rolling resistance) to account for the effect of particle shape angularity, e.g. resistance to relative rotations due to particle interlocking, which is encountered in real irregularly shaped grains. We have DEM output data for every grain over 299 equilibrium states, spanning all regimes of the loading history that culminates in the limit state where the sample fails by strain localization. A single fully developed shear band forms, which is inclined approximately along the main forward diagonal (from bottom left to top right corner of each sample) with a global anticlockwise rotation. The shear band governs system dynamics in the limit state: distinct fluctuations manifest in the stress ratio, indicative of stick-slip dynamics. During stick or rise in stress ratio, the dominant mesoscale mechanism inside the shear band is jamming whereby clusters of particles form new stable, densely-connected configurations embodying force chains. During the ensuing slip or drop in stress ratio, the dominant mesoscale mechanism inside the shear band is unjamming whereby force chains fail by buckling. Force

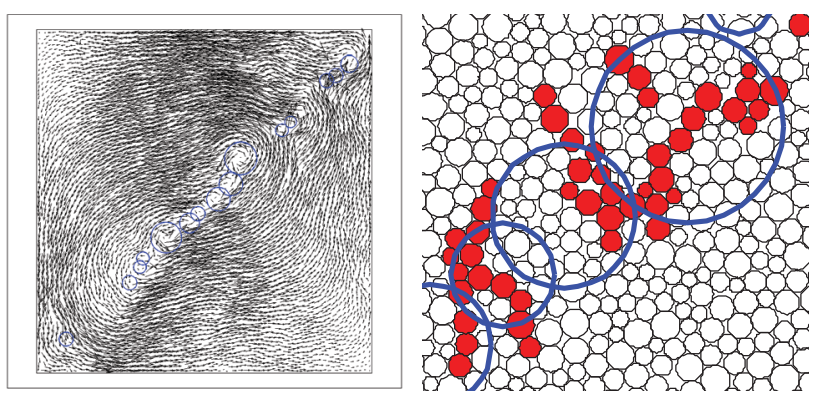

Figure 1. A representative displacement vector field during unjamming in the limit state showing the shear band (left); a closeup view of buckling force chains shown here by the red colored grains (right). Displacement vectors in vortices, identified and characterized in [8], are highlighted by the blue circles.

chain buckling is accompanied by, and induces, local grain rearrangements that are highly nonaffine, dilatant, and dissipative $[9,10]$. These rearrangements manifest a pattern of motion that is rich in vortices - and mediates the relative rigid-body motion of the regions on either side of the shear band, as shown in Figure 1.

\section{Methods}

Let $N$ be the total number of grains in the sample, and $\left(x_{i}(t), y_{i}(t), z_{i}(t)\right)$ be the location of grain $i$, where $i=$ $1,2,3, \ldots, N$, at each equilibrium state $t$. For the data described in Section 2, $N=5098$. The displacement of grain $i$ is $d_{i}(t)=\left(\Delta x_{i}(t), \Delta y_{i}(t), \Delta z_{i}(t)\right)$, where

$$
\begin{aligned}
\Delta x_{i}(t) & =x_{i}(t)-x_{i}(t-1) \\
\Delta y_{i}(t) & =y_{i}(t)-y_{i}(t-1) \\
\Delta z_{i}(t) & =z_{i}(t)-z_{i}(t-1)
\end{aligned}
$$

At each equilibrium state $t$, we construct a graph $G(r)$ in the displacement-state-space (DSS) where the nodes represent the grains. Two nodes, $i$ and $j$, share a link if the difference in their displacements satisfies

$$
\left|d_{i}(t)-d_{j}(t)\right| \leq r
$$

Thus, two nodes are connected if their Euclidean distance in DSS is at most $r$, where $r>0$ is a free parameter (Figure 2). We then study how $G(r)$ changes as $r$ increases from 0 by a constant and small increment $\delta$. When $r$ is small, only a few links exist: $G$ is sparsely connected and is a multi-component graph. As $r$ increases, more and more nodes are connected, so that eventually $G$ becomes a single-component graph. Let $S(r)$ be the size of the largest connected component in $G(r)$, the so-called giant component of $G(r)$. The size of $S$, given by the total number of nodes in $S$, increases from 0 to its maximum possible value of $N$. In Figure 2, $N=27, G(r)$ is an 8-component graph (sizes $10,7,4,2,1,1,1,1)$ and $S(r)$ is size 10; but increasing $r$ to $r+R$ (constant $R>>$ ) would lead to $G(r)=S(r)$ of size 27 because of the bridge nodes. 


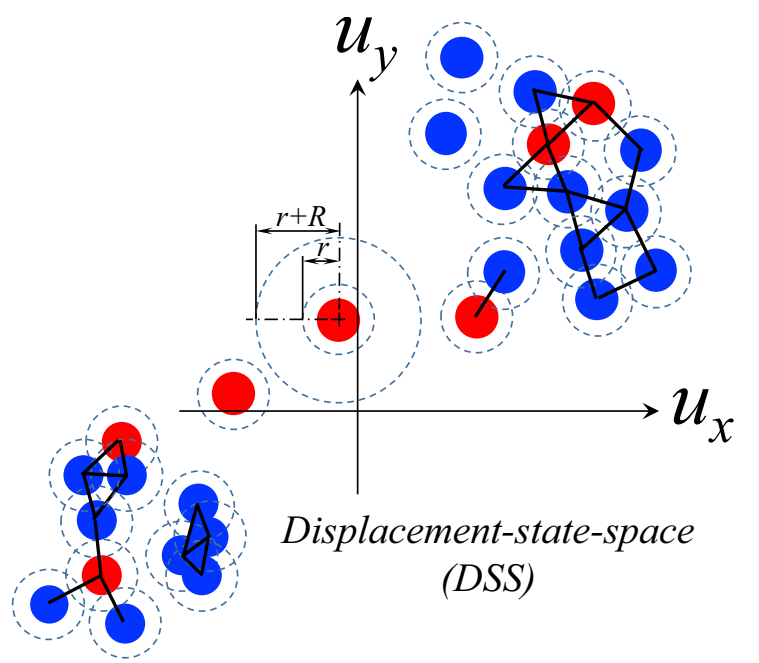

Figure 2. Schematic plot of the method for constructing $G(r)$ for $N=27$ nodes. Each node is located at its corresponding grain displacement vector $\left(u_{x}, u_{y}\right)$. For a given radius $r$, two nodes within a distance $r$ (radius of dashed circle) in DSS are connected by a link. Red nodes act as a bridge between the two largest communities of sizes 10 (top-right) and 7 (bottom-left), respectively.

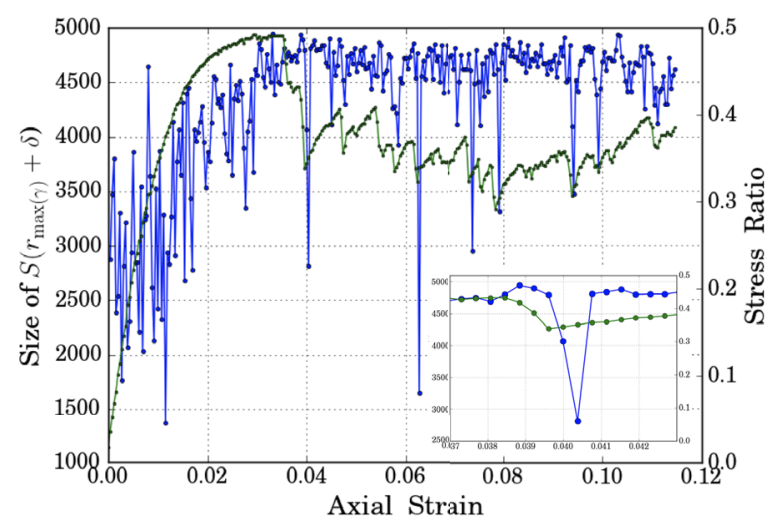

Figure 3. Evolution with strain of the size of $S\left(r_{\max (\gamma)}\right)$ and stress ratio. The significant drops in the critical state regime occur during jamming cycles: inset enlarges the rise in stress after $\approx 0.04$ axial strain.

\section{Results}

In percolation theory, a key quantity is the order parameter, which is used to characterize the degree of order of the system. The order parameter is a normalized quantity between 0 and 1 . A system is termed disordered (ordered) if the corresponding order parameter is close to 0 (1). Moreover, a percolation process is called continuous (explosive) if the order parameter is a continuous (discontinuous) function of the microscopic parameter $r$. Order parameters can be defined in various ways as needed. The most common order parameter in percolation theory is the probability that a selected node belongs to the giant component $S(r)$. Given that $r>0$, this probability can be expressed as

$$
p(r)=\frac{S(r)}{N},
$$

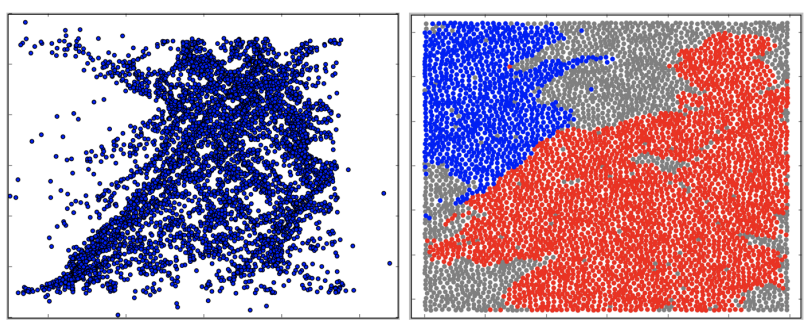

Figure 4. Pre-failure regime. Scatter plot of displacements in DSS at the axial strain interval of [0.0008,0.0012] (left). Member nodes of the different components in $G(r)$ just before percolation transition $S\left(r_{\max (\gamma)}-\delta\right)$, mapped back into the physical domain of the sample: members of the largest and second largest connected components are colored red and blue, respectively, with the rest in grey (right).

with $S(r)$ the size of the largest connected component in $G(r)$. When $r$ is small, $G(r)$ is sparsely connected: $p(r)$ is close to zero. When $r$ is large, $G(r)$ is densely connected: $p(r)$ is close one.

For finite systems, the function $p(r)$ can contain distinct jump discontinuities, $\gamma(r)>\gamma(r-\delta)$, as $r$ is increased at small and fixed increment $\delta$, where $\gamma(r)=[p(r)-p(r-\delta)] / \delta$. The evolution with strain of the size of $S\left(r_{\max (\gamma)}\right)$ where $r=r_{\max (\gamma)}$ is where $\gamma$ is maximum is shown in Figure 3. We see that $S\left(r_{\max (\gamma)}\right)$ increases initially but becomes almost invariant in the limit state. This trend is consistent with the distribution of displacements in the DSS as shown both prior to and during failure in Figures 4(left) and 5(left), respectively. The drops in the size of $S\left(r_{\max (\gamma)}\right)$ occur during jamming when grains lock up and their motions momentarily subdued.

The corresponding visualisations in Figures 4(right) and 5(right) show the locations of the member nodes of the different components in $G(r)$ just before the percolation transition, at $r=r_{\max (\gamma)}-\delta$, in the physical domain of the sample. Two key features of the displacement-statespace in Figure 5 distinguish it from that in Figures 4. First, there are two distinct communities of nodes representing the two oppositely moving rigid-body parts of the sample, whenever the shear band unjams. Second, the nodes in the shear band mediate, and thus enables motion transfer, between the two communities.

Finally, we see from Figure 6 that the transition becomes explosive in the limit state - when a fully developed shear band inhabits the sample. Here we observe a prolonged period when the size of the giant component $S(r)$ is almost invariant as $r$ is increased incrementally by an amount $\delta$, where $\delta$ is very small relative to system size. This prolonged invariance of $S(r)$ is indicative of the wide separation of the largest components (network communities) in $G(r)$ in the limit state. The shear band, however, acts as bridge nodes: compare the depiction in Figure 2 and the distribution of the nodes in DSS in Figure 5. Due to the shear band nodes, we observe a sudden and large jump increase in $p(r)$ to just below 1 , indicating an explo- 


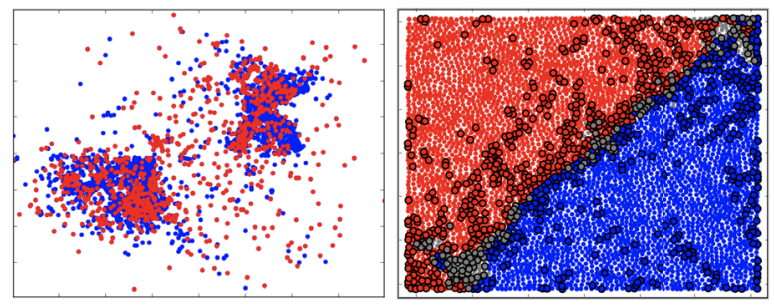

Figure 5. Failure regime. Scatter plot of displacements in DSS at the axial strain interval of [0.0388,0.0392] (left): here nodes highlighted in red correspond to those grains with the highest rotations (top 20\%) which are mainly in the shear band. Member nodes of the different components in $G(r)$ just before explosive percolation $S\left(r_{\max (\gamma)}-\delta\right)$, mapped back into the physical domain of the sample: members of the largest and second largest connected components are colored red and blue, respectively, with the rest in grey (right). Grains with the highest rotations are highlighted by the black outlined circles.

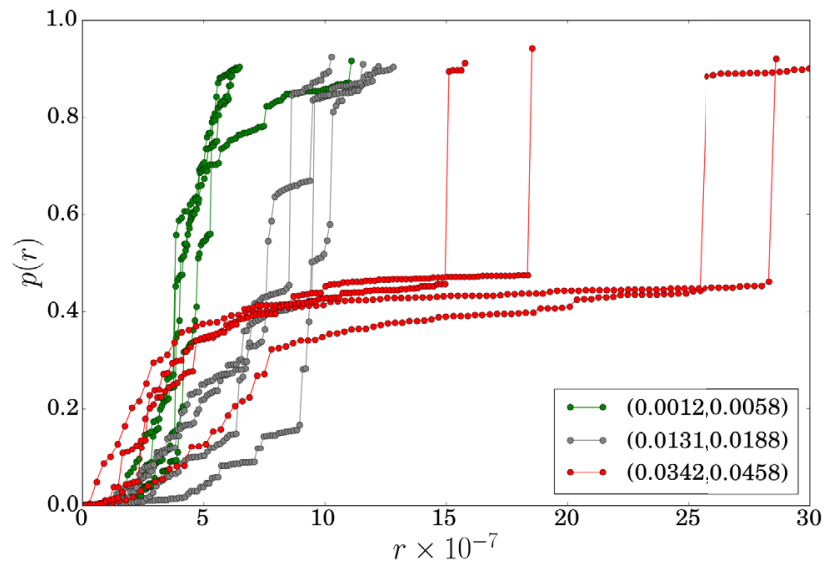

Figure 6. Evolution of $p(r)$ versus $r$ in the lead up to and during the limit state.

sive growth in the size of $S(r)$ to near its maximum value just after the explosive percolation transition.

\section{Conclusion}

The process of percolation in graphs embodying information on individual grain kinematics is investigated to determine the existence of a possible critical transition in the underlying microstructure and dynamics of a dense granular material as it evolves towards the so-called critical state regime. We find that the presence of strain localization is precisely that which renders this limit state critical when viewed from the perspective of a topological-dynamical phase transition. Moreover the transition is consistent with that of an explosive percolation. We attribute this to the emergence of self-organized collective motion, which result in increasingly isolated kinematical communities that are mediated by the shear band. This finding casts light on the dual and opposing roles of the shear band: a mechanism that creates subdivisions in the sample during unjamming events, while simultaneously acting as a mechanical link that transfers motion through such subdivisions moving in relative rigid-body motion. A full analysis of the percolation properties of $G(r)$ in the lead up to and during the limit state, including the evolution of the critical percolation transition is reported in [11].

\section{Acknowledgements}

The authors acknowledge the support of the Australian Research Council (DP120104759), the U.S. Air Force (AFOSR 15IOA059), and the U.S. Army Research Office (W911NF-11-1-0175, W911NF-15-1-0527).

\section{References}

[1] A. Aharony, D. Stauffer, Introduction To Percolation Theory (Taylor \& Francis, 2003)

[2] Rozenfeld, H. D., Gallos, L. K., Makse, H. A., Eur. Phys. J. B 75, 305 (2010)

[3] R.K. Pan, M. Kivelä, J. Saramäki, K. Kaski, J. Kertész, Phys. Rev. E 83, 046112 (2011)

[4] R.M. D’Souza, J. Nagler, Nature Physics 11, 531 (2015)

[5] B. Brady, E. Brown, Rock Mechanics: For underground mining (Springer Netherlands, 2007)

[6] C. Hall, W. Hoff, Water Transport in Brick, Stone and Concrete (Taylor \& Francis, 2002)

[7] D. Achlioptas, R.M. D’Souza, J. Spencer, Science 323, 1453 (2009)

[8] A. Tordesillas, S. Pucilowski, Q. Lin, J.F. Peters, R.P. Behringer, Journal of the Mechanics and Physics of Solids 90, 215 (2016)

[9] A. Tordesillas, Philosophical Magazine 87, 4987 (2007)

[10] A. Tordesillas, S. Pucilowski, D.M. Walker, J.F. Peters, L.E. Walizer, International Journal for Numerical and Analytical Methods in Geomechanics (2014)

[11] Z. Zhou, A. Tordesillas, in preparation (2017) 